\title{
CONSTRUCCIONES POSESIVAS DEL MAPUZUGUN. UNA APROXIMACIÓN TIPOLÓGICA ${ }^{1}$ \\ POSSESSIVE CONSTRUCTIONS IN MAPUZUGUN. A TYPOLOGICAL APPROACH
}

\author{
ALDO OLATE \\ Universidad de La Frontera \\ aldo.olate@ufrontera.cl \\ RODRIGO BECERRA \\ University of Alberta \\ rbecerra@ualberta.ca \\ FERNANDO ZÚNIGA \\ Universität Bern \\ fernando.zuniga@isw.unibe.ch \\ JAQUELINE CANIGUAN \\ Universidad de La Frontera \\ jacqueline.caniguan@ufrontera.cl
}

\section{RESUMEN}

Este trabajo explora las construcciones posesivas del mapuzugun desde la lingüística tipológica contemporánea. A partir de los datos recogidos y previa discusión bibliográfica, se establecen los esquemas posesivos prototípicos del mapuzugun y sus variaciones. Finalmente, se propone una clasificación tipológica de las construcciones de la lengua de acuerdo con la posesión nominal, predicativa y externa, concluyendo con algunas reflexiones generales sobre el fenómeno de la posesión en mapuzugun.

Palabras clave: Construcciones posesivas, posesión interna, predicativa y externa, tipología lingüística, mapuzugun.

${ }^{1}$ El presente trabajo se realiza gracias al apoyo del proyecto FONDECYT 1180071: "TAM, evidencialidad, fenómenos asociados a la valencia verbal y la codificación de eventos complejos en el castellano de contacto hablado por bilingües mapuche/castellano. Un estudio comparativo del sistema verbal". 


\section{ABSTRACT}

This paper explores possessive constructions in Mapuzugun from the perspective of current linguistic typology. Based on published sources and fieldwork data, we establish the prototypical possession schemes and their variation in Mapuzugun. Finally, we propose a typological classification of the constructions surveyed and advance some general reflections upon this phenomenon in the language studied.

Keywords: Possessive constructions, internal, predicative, and external possession, linguistic typology, Mapudungun.

Recibido: 03.05.2018. Aceptado: 20.11.2018.

\section{PRESENTACIÓN}

E

n su gramática de la lengua mapuche de 2006, Zúñiga presenta las características de la estructura posesiva del mapuzugun. Allí, a través de una nota comparativa, señala que en las lenguas del mundo existen cuatro estrategias básicas para indicar posesión atributiva: (a) marcar el poseedor, (b) marcar lo poseído, (c) marcar ambos elementos de la estructura y (d) no marcar ninguno explícitamente. En esta tipificación, el mapuzugun se caracteriza por marcar la entidad poseída (tipo b), estrategia que lo asemeja al húngaro y lo diferencia de lenguas como el castellano y el inglés. Sin embargo, se diferencia del húngaro por la manera en cómo marca el poseído. Mientras esta última lengua marca la entidad poseída por medio de un sufijo personal, el mapuzugun lo hace por medio de una marca independiente antepuesta.

En el presente trabajo retomamos la perspectiva de Zúñiga (2006) para extenderla y actualizarla, indagando en otras construcciones posesivas de la lengua mapuche. Si bien diversos subsistemas del mapuzugun ya han sido descritos en la literatura, el ámbito de la posesión no ha sido abordado con suficiente detalle. Los motivos pueden ser diversos, entre ellos, el desarrollo reciente de los estudios tipológicos y, específicamente, de la posesión. En este contexto, el objetivo de este trabajo es describir las estructuras posesivas del mapuzugun hablado en el área de la Araucanía (Chile) y caracterizarlas desde un marco tipológico.

La descripción que presentamos es relevante para enriquecer el conocimiento de las lenguas indoamericanas y surandinas, y el conocimiento tipológico en general. En primer lugar, permite situar la lengua mapuche en el marco de la tipología de las estructuras posesivas; en segundo lugar, pone a disposición insumos para establecer posibles relaciones lingüísticas areales $\mathrm{y}$, finalmente, provee datos que enriquecen el conocimiento de las propias tipologías propuestas.

En función de lo anterior, esta exposición se organiza de acuerdo a los siguien- 
tes temas: (1) la posesión en general; (2) revisión de estudios sobre estructuras posesivas en el mapuzugun; (3) la investigación particular, lugar donde presentamos la muestra, los criterios de análisis y la descripción lingüística de los esquemas que se encuentran en nuestro corpus.

\subsection{Aspectos generales de la posesión}

La posesión es una noción abstracta que establece la relación entre un Poseedor y un Poseído $^{2}$. Heine (1997) sugiere que es una categoría lingüística universal que tiene formas convencionalizadas de expresión en todas las lenguas, aunque es también sensible a especificaciones culturales. En un plano semántico, la posesión cubre varios tipos de relaciones, cuyas realizaciones en las gramáticas de las lenguas pueden adoptar diversas formas. Así, por ejemplo, del espectro de relaciones expresadas por medio de la posesión podemos nombrar: (1) propiedad ('la casa de Rodrigo'), (2) parte-todo ('la ventana de la pieza'), (3) relaciones de parentesco ('la hija de Aldo'), (4) relación atributiva ('el humor de Fernando'), (5) establecimiento de orientación o localización ('el costado de la calle'), y (6) asociación ('el dentista de Jaqueline') (Dixon, 2010). La variación inter-lingüística de este tipo de construcciones es evidente y concierne al tipo de nombre (i.e. persona, animal o idea) que funciona como Poseedor o Poseído (Dixon, 2010).

Existen varios trabajos que abordan el fenómeno de la posesión en lenguas indoamericanas desde un enfoque descriptivo y tipológico (Fernández Garay, 2004, Álvarez, 2012; Pamies, 2004; Oquendo, 2007; Álvarez 1996; Álvarez y Muchembled 2015). En este estudio describimos tres tipos de estructuras posesivas del mapuzugun: (a) posesión nominal (o atributiva), (b) posesión predicativa y (c) posesión externa. La discusión se realiza siguiendo las perspectivas de Dixon (2010), Nichols y Bickel (2013a; 2013b), Bickel y Nichols (2013), Stassen (2009, 2013), y Aikhenvald $(2013,2015)$.

\subsection{Las estructuras posesivas desde la perspectiva tipológica}

La gran mayoría de los estudios sobre construcciones posesivas se enfocan en lo que se reconoce como posesión atributiva o nominal (interna) y posesión predicativa. Un tercer tipo de posesión -más compleja- es la posesión externa, que tiene que ver con cambios en la valencia verbal y posibles procesos de aplicativización. A continuación se desarrollan breves discusiones sobre estas tres maneras de expresar la posesión desde la perspectiva tipológico-comparativa.

${ }^{2}$ Adoptamos la convención de escribir los roles semánticos de la posesión con mayúsculas. 


\subsubsection{La posesión nominal}

En esta sección se presentan dos tradiciones de descripción de las construcciones posesivas nominales (PN); en primer lugar, la desarrollada al alero de la escuela tipológica del Instituto Max Planck (Bickel y Nichols, 2013; Nichols y Bickel, 2013a y b) y, en segundo lugar, la desarrollada por Dixon (2010) y Aikhenvald $(2013,2015)$.

De acuerdo con Nichols y Bickel (2013b), las lenguas codifican la posesión de diversas formas, pudiendo tener más de una para expresar dicho contenido. Así, muchas lenguas tienen sistemas binarios de clasificación posesiva, en tanto unas pocas tienen sistemas más complejos. En inglés, por ejemplo, esta relación puede expresarse de dos maneras: el genitivo sajón y el normando (v.gr. John's house y The Houses of Parliament, respectivamente), cuya distribución está influida principalmente por los rasgos semánticos del Poseedor. Por el contrario, hay lenguas que generan oposiciones entre dos formas de marcas posesivas condicionadas por las propiedades del elemento que opera como Poseído en la construcción, como en el ejemplo siguiente (1):

(1) Diegueño de Mesa Grande, familia hokana (Nichols y Bickel, 2013b)
a. P-ataly
1sG-madre
b. $\quad P \partial-n^{y}-e w a:$
'mi madre'
1SG-ALIENABLE ${ }^{3}$-casa
'mi casa'

Nichols y Bickel (2013b) proponen la etiqueta de "clasificación posesiva" para dar cuenta de la tipificación de los sustantivos, los que, en tanto núcleo de la construcción posesiva, pueden dividirse en dos grupos: separables (alienables) e inseparables (inalienables). Bickel y Nichols (2013) discuten casos de posesión por medio de núcleo marcado. Así, en el caso de la llamada posesión obligatoria, algunos nombres requieren flexión para poder ser utilizados en el discurso. Aquellos nombres que la exigen son clasificados como nombres poseídos obligatoriamente y se discuten en el marco de la distinción alienable-inalienable.

En cuanto a las formas que instancian la posesión, pueden corresponder a un

${ }^{3}$ Las abreviaturas utilizadas en este trabajo son las siguientes: $1=$ primera persona; $2=$ segunda persona; 3 = tercera persona; $\mathrm{A}=$ sujeto transitivo; $\mathrm{CC}=$ complemento copulativo; $\mathrm{ABL}=$ ablativo; $\mathrm{ACUS}$ = acusativo; $\mathrm{ACT}=$ activo; $\mathrm{ANIM}=$ animado; $\mathrm{APLI}=$ aplicativo; $\mathrm{ART}=$ artículo; $\mathrm{CL}=$ clasificador $\mathrm{CNSTR}=$ status constructus; $\mathrm{COP}=$ cópula; $\mathrm{DECL}=$ declarativo; $\mathrm{D}=$ Poseído; $\mathrm{DEF}=$ definido; $\mathrm{DEM}=$ demostrativo; $\mathrm{DET}=$ determinante; $\mathrm{DIR}=$ directa; $\mathrm{DISC}=$ partícula discursiva; $\mathrm{DU}=$ dual; $\mathrm{FEM}=$ femenino; $\mathrm{GEN}=$ genitivo; $\mathrm{HAB}=$ habitual; IMP = imperativo; IND = modo indicativo; INTR = intransitivo; INV = inversa; MASC $=$ masculino MCN $=$ marca de clase nominal NEG = polaridad negativa; $\mathrm{NOM}=$ nominativo; $\mathrm{O}=$ objeto PAS = pasiva; PERT $=$ pertensivo $; \mathrm{PL}=$ plural; $\mathrm{POS}=$ posesivo $; \mathrm{POSP}=$ posposición ; PRES $=$ presente; $\mathrm{R}$ = Poseedor; $\mathrm{RFX}=$ reflejo-recíproco; $\mathrm{S}$ = sujeto intransitivo; $\mathrm{SG}=$ singular; $\mathrm{TOP}$ = tópico. 
mismo tipo de clase morfológica o a distintas clases, distribuidas de acuerdo con la taxonomía de los sustantivos. De este modo, por ejemplo, una clase de sustantivos puede tomar afijos posesivos mientras que otra clase puede tomar pronombres posesivos (2):

(2) Warndarang, familia Mangarrayi-Maran (Australia) (Nichols y Bickel, 2013b)
a. $n g-b a b a$
b.
wu-radburru
ngini
1-padre
'mi padre', 'nuestro padre'

NCM-país
'mi país'

Por último, Nichols y Bickel (2013a) consignan las cuatro estrategias más frecuentes de la marcación morfológica en la estructura posesiva nominal: (i) marca (“posesiva”) en el núcleo; (ii) marca ("genitiva”) en el dependiente; (iii) doble marca; y (iv) cero. Las otras dos estrategias identificadas son (v) marca libre/flotante (cuando se posiciona en relación con los límites del sintagma en lugar del núcleo o el dependiente) y (vi) marca en el dependiente migrada hacia el núcleo (cuando pronombres personales/posesivos se transforman en clíticos del núcleo).

Un acercamiento distinto al análisis de este dominio es el propuesto por Dixon (2010) y Aikhenvald (2013), quienes conciben la posesión a partir de las relaciones semánticas que pueden ser cubiertas por una construcción PN. Los componentes de este tipo de construcciones son el Poseedor (R), que puede ser actualizado por nombres, pronombres o frases nominales y el Poseído (D), que puede ser un nombre o frase nominal (Dixon, 2010).

Aikhenvald (2013) sostiene que ciertas categorías lingüísticas muestran correlaciones con valores culturales, jerarquías sociales y sus conceptualizaciones. Así, el género, las clases de sustantivos y los clasificadores tienden a reflejar estereotipos culturales y patrones de percepción, por lo que el contacto entre la cultura y la lengua puede afectar el tratamiento de las relaciones posesivas y, por consiguiente, la categorización del Poseedor y Poseído. En este ámbito, Dixon (2010) y Aikhenvald (2013) muestran tres tipos de relaciones de posesión nucleares que son recurrentes en su mirada inter-lingüística: (A) propiedad, (B) parte-todo, y (C) relaciones de parentesco (consanguíneas y de afinidad). Las lenguas pueden usar una misma construcción para $\mathrm{A}, \mathrm{B}$ y $\mathrm{C}$, cubrir A y B con una construcción y $\mathrm{C}$ con otra, o contar con construcciones distintas para $\mathrm{A}, \mathrm{B}$ y $\mathrm{C}$.

Estas relaciones pueden seguir el principio de la iconicidad gramatical (Haspelmath, 2008), ya que tanto B como $\mathrm{C}$ implican una fuerte relación entre los referentes, lo que se traduce en que, por ejemplo, la 'parte' difícilmente pueda ser expresada sin el 'todo', o que en las relaciones de parentesco uno no pueda ser definido sino en relación al otro. Este principio está en la base de la posesión alienable e inalienable, y cuando una lengua las exhibe, las codifica de formas distintas. Así, 
la posesión inalienable se presenta con menos marcas formales que la alienable, tendiendo esta última a ser expresada por medio de estructuras analíticas. A nivel morfológico, la marca puede ser un segmento más largo que el que se utiliza para la posesión inalienable (Dixon, 2010; Aikhenvald, 2013).

Aikhenvald (2013) reconoce cinco estrategias para marcar la relación de posesión: (i) orden de las palabras en la frase nominal; (ii) marca ("pertensiva") sobre el Poseído ${ }^{4}$; (iii) marca ("genitiva") sobre el Poseedor; (iv) marcas sobre ambos, y (v) marca independiente. La marca pertensiva puede ser invariable y general, como el status constructus de las lenguas semíticas y oceánicas, o variable y específica, como los afijos posesivos de muchas lenguas (ver más abajo).

Aikhenvald $(2013,2015)$ coincide con Dixon (2010) en que es necesario conocer tanto la naturaleza del Poseedor como la del Poseído para entender el tipo de relación que se establece y las alternativas de marcación en la lengua. Como muestra Dixon (2010), en el caso de la estrategia de la yuxtaposición se pueden presentar dos tipos de organización: una donde $\mathrm{R}$ precede a D (3-4) y otra donde se da el orden opuesto (D R) (5):

(3) Angami, familia sinotibetana (Dixon, 2010: 267)
a. $\mathrm{R} \quad \mathrm{D}$
$\hat{a} \quad z \bar{e} u ̀$
b. $\mathrm{R} \quad \mathrm{D}$
$1 \mathrm{SG} \quad$ amigo
$m \bar{I} z \bar{\partial} \quad p h i$
'mi amigo'
mesa pierna
'pata de la mesa'

Ejemplos de marcación morfológica ya fueron presentados en (1) y (2). En (4) se observa cómo, en la lengua yagua, si R no es realizado por un sustantivo o un sintagma nominal, un prefijo personal posesivo se añade a D (4a), y en caso contrario solo opera la yuxtaposición (4b):

(4) Yagua, familia peba-yagua (Perú) (Dixon, 2010: 272)
a. $\mathrm{R}-\mathrm{D}$
b.
$\mathrm{R}$
$\mathrm{D}$
sa-rooriy
3sG.R-casa
'su casa'

Tomáása rooriy
Tomasa casa
'la casa de Tomasa'

Otro par de estrategias pueden observarse en tialo (5): de manera similar al yagua, cuando $\mathrm{R}$ no es instanciado por un sintagma nominal, $\mathrm{D}$ recibe un sufijo 268).

${ }^{4}$ El término pertensive 'pertensivo', derivado del latín pertinère 'pertenecer', es de Dixon (2010: 
En este ejemplo se observa que cuando $\mathrm{R}$ es un pronombre personal, se utiliza una forma posesiva del mismo que antecede a D (6a); si $\mathrm{R}$ es un nombre, se emplea una marca posesiva independiente entre $\mathrm{D}$ y $\mathrm{R}$, ya sea $a$, como en (6b) (si R es humano) o $d$, como en (6c) (si R es no humano).

\subsubsection{Posesión alienable versus posesión inalienable}

Así como existen diversas marcas para la construcción posesiva de acuerdo con la naturaleza del Poseedor, también las hay según la naturaleza del Poseído. Dixon (2010) estudia las bases semánticas de los nombres que funcionan como D en una construcción PN, identificando dos conjuntos de nombres que contraen dicha función: el grupo uno o "inalienable", que define una fuerte relación con el Poseedor, y el grupo dos o "alienable", que considera otros tipos de relación. A través de esta clasificación, da cuenta de la tendencia que exhiben los nombres en ocho lenguas indígenas del mundo. La clasificación se asocia con las relaciones de posesión nucleares señaladas por Aikhenvald (2013), y es extendida a algunos tipos de relaciones subsidiarias, a saber: atributiva, de orientación y localización, y de asociación.

La relación parte-todo (tipo B) se presenta en siete lenguas dentro del conjunto uno. Según Dixon (2010), esta relación tiene siete subvariedades: partes externas del cuerpo, partes internas, genitales, fluidos corporales, partes de animales, partes de plantas, y partes de artefactos y otros objetos. Por otro lado, la distribución de las relaciones de parentesco (tipo $\mathrm{C}$ ) es menos diversa que la del tipo $\mathrm{B}$, pues aparecen sólo en cinco lenguas dentro del conjunto uno. La variación de los nombres que integran el conjunto uno es amplia, y la etiqueta "inalienable" pareciera aplicarse individualmente en cada lengua. Es decir, la etiqueta no presenta contenido semántico invariable inter-lingüísticamente para una clase gramaticalmente definida.

Dixon (2010) muestra diversas formas en que puede contrastar la expresión de la posesión alienable versus inalienable. En algunos casos, la posesión alienable puede expresarse de la misma forma que la inalienable, salvo por la inclusión de una marca pertensiva invariable en la forma alienable (7):

(7) Guajiro, familia arawak (Dixon, 2010: 286)
a. R-D
b. R-D
ta-ye:
ta-kulu:t-se
1sG.R-lengua
1sG.R-ropa-PERT
'mi lengua' (inalienable)
'mi ropa' (alienable)

En este sentido, se observa que la expresión de la marca para la posesión alie- 
nable es, generalmente, más larga que la usada para la posesión inalienable. Una manera distinta es el empleo de un clasificador, usado solamente en la posesión alienable (8):

(8) Panare, familia caribe (Dixon, 2010: 288)

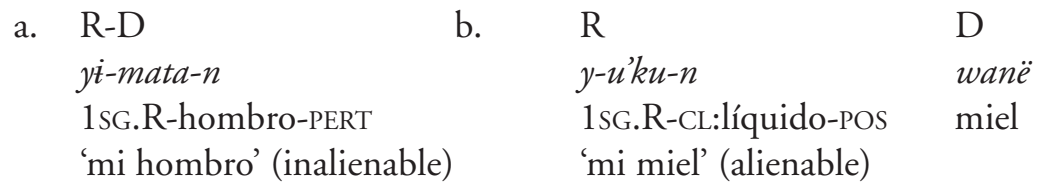

Por último, se ha descrito el simple uso de aposición para expresar posesión alienable, lo que contrasta con el uso de marcación explícita ("overt marking") en la construcción alienable (9):

(9) Lango, familia nilótica oriental (Dixon, 2010: 288)
a. $\mathrm{D} \quad \mathrm{R}$
léb lócà -
b.
$\mathrm{D}$
$\mathrm{R}$
lengua hombre
gwôkk a lócà-
'la lengua del hombre' (inalienable)
perro pOs hombre 'el perro del hombre' (alienable)

En este último caso, la partícula atributiva $a$ aparece entre D y R, lo que contrasta con el uso de aposición para la expresión de la posesión inalienable.

\subsubsection{La posesión predicativa}

Stassen $(2009,2013)$ desarrolla la noción de posesión predicativa (PP), entendiéndola como relacional, asimétrica y prototípicamente alienable. Su definición supone la existencia de contacto permanente entre dos elementos, y el control del Poseedor (R) sobre el Poseído (D) (Álvarez y Muchembled, 2015: 99-100; Muchembled, 2014). Stassen (2013) reconoce cuatro estrategias por medio de las cuales se codifica este tipo de posesión: (i) posesión con verbo 'tener' o 'pertenecer'; (ii) posesión oblicua (posesiva locativa y posesiva genitiva); (iii) posesión con tópico: y (iv) posesión conjuntiva 5 .

De estas cuatro estrategias, la primera codifica la relación entre $\mathrm{R}$ y $\mathrm{D}$ en la forma de una construcción transitiva, lo que se traduce en que el SN Poseedor puede

${ }^{5}$ Dixon (2010) agrupa estas construcciones en tres tipos: (i) la posesión con 'tener', de naturaleza transitiva; (ii) la posesión con cópula, que se manifiesta a través de un caso oblicuo o adposición; y (iii) las construcciones posesivas con el verbo 'existir'. En este apartado seguiremos la propuesta de Stassen $(2009,2013)$ por su mayor grado de especificación. 
operar como sujeto y el SN Poseído como objeto directo (u objeto primario) de un verbo equivalente a 'tener'. En muchos casos, este verbo deriva de verbos que indican control físico o manipulación ('tomar', 'agarrar', 'retener' o 'llevar', como en el origen del verbo castellano tener < tenère 'sostener, agarrar, tener'). Un ejemplo de este tipo de PP lo tenemos en (10), donde el Poseído se interpreta como objeto directo del verbo 'tener':

(10) Latín, familia indoeuropea

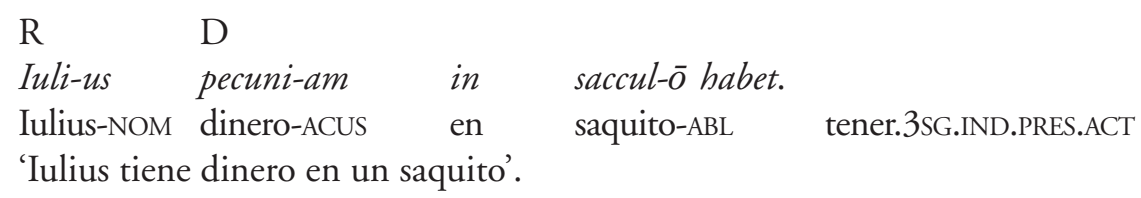

La primera estrategia tiene un segundo subtipo, que distribuye los roles semánticos de manera opuesta. En esta el SN Poseedor opera como objeto directo o indirecto y el SN Poseído como sujeto, lo que ocurre en las construcciones castellanas con el verbo 'pertenecer'. En suma, en ambos subtipos las funciones argumentales son distintas, pues en el primero el Poseedor es el sujeto $(R=A)$ y el Poseído es el objeto $(D=O)$, mientras en el segundo el Poseído es sujeto $(D=A)$ y el Poseedor es el objeto $(\mathrm{R}=\mathrm{O})$.

Los otros tres mecanismos (posesión oblicua, con tópico y conjuntiva) tienen en común el rasgo de la intransitividad. En todas ellas la construcción posesiva tiene la forma básica de una oración existencial, el predicado puede tener un valor locativo o existencial, y la traducción puede corresponder a 'estar con', 'estar en' o 'estar allí. La diferencia entre dichas construcciones tiene relación con la manera en que codifican el Poseedor o lo Poseído (Stassen, 2013).

En la posesión de construcción oblicua se observa que el Poseído tiene el papel de sujeto gramatical mientras que el Poseedor es constituido por una forma oblicua con dos subtipos (dependiendo del tipo de relación seleccionada): una construcción posesiva con el oblicuo locativo, o una construcción posesiva genitiva. El primer caso se muestra en (11), en el que el morfema -dur expresa el valor locativo adherido al Poseedor, mientras que el segundo subtipo se muestra en (12), en que el Poseedor se expresa por medio de un modificador genitivo que antecede al Poseído (Stassen, 2013).

(11) Mongol escrito, familia altaica (Stassen, 2013)

Na-dur morin
1SG-en caballo


(12) Avar, familia nakh-daghestaniana (Cáucaso) (Stassen, 2013)

$\begin{array}{lll}\text { Dir } & \text { mašina } & b \text {-ugo. } \\ \text { 1SG.GEN } & \text { automóvil } & \text { III-ser.PRES }\end{array}$

'Tengo un auto'. (lit. 'es un automóvil mío')

La construcción posesiva con tópico tiene en común con las anteriores la expresión del Poseído como sujeto gramatical (relacionada al predicado existencial), lo que la distingue es su codificación del Poseedor como el tema de la oración. La estructura nominal que funciona como el escenario de la oración se entiende como el marco discursivo, restringiendo el valor de verdad de la oración que sigue (Stassen, 2013). Esto lo podemos observar en (13):

(13) Tondano, familia austronésica (Stassen, 2013)

$\begin{array}{llllll}\text { Si } & \text { tuama } & \text { si } & \text { wewean } & \text { wale } & \text { rua. } \\ \text { ANIM.SG } & \text { hombre } & \text { TOP } & \text { existir } & \text { casa } & \text { dos } \\ \text { 'El hombre tiene dos casas'. (lit. 'en cuanto al hombre, hay dos casas') }\end{array}$

Finalmente, en la construcción posesiva conjuntiva el Poseedor es el sujeto gramatical y la estructura nominal del Poseído va acompañada de una marca que no tiene valor locativo ni topicalizador. El valor de la marca tiene su origen en la simultaneidad interclausal, por lo que se encuentran marcadores con origen en adverbios ('también'), en conjunciones subordinantes ('cuando'/'mientras') o en conjunciones coordinantes (' $y$ '). La estrategia prototípica de esta modalidad es el uso del comitativo ('con') sobre la estructura nominal del Poseído. Un ejemplo de esta modalidad se observa en (14):

(14) Daga, familia daga (Papúa Nueva Guinea) (Stassen, 2013)

Orup da agoe den
hombre uno esclavo con/también
'Un hombre tiene un esclavo.'

Por su parte, Aikhenvald (2013) propone una taxonomía relativamente simple de las estrategias formales empleadas para expresar la posesión predicativa: (i) construcciones con verbos de propiedad (incluyendo aquellos como 'tener' y aquellos como 'pertenecer'); (ii) construcciones copulativas y cláusulas sin verbo; y (iii) construcciones con derivaciones posesivas (donde la posesión se expresa mediante afijos derivacionales). 


\subsubsection{La posesión externa}

La posesión externa (PE) o separación del Poseedor se caracteriza por ser una construcción en la que la relación semántica Poseedor-Poseído se expresa por medio de la codificación del Poseedor $(\mathrm{R})$ como argumento sintáctico de la relación gramatical establecida por el verbo y en un constituyente separado del que contiene al Poseído (D). R puede ser expresado como sujeto, objeto directo u objeto indirecto, dependiendo del tipo de lengua (Payne y Barshi, 1999). En algunos idiomas, además de ser expresado como argumento sintáctico, $\mathrm{R}$ puede codificarse simultáneamente por medio de un pronombre o afijo pronominal interno de la frase nominal que contiene a D. En estos casos, igualmente $\mathrm{R}$ es expresado como argumento, y puede serlo de construcciones intransitivas, transitivas o ditransitivas (Payne y Barshi, 1999).

Las construcciones con PE presentan un participante adicional en la estructura eventiva, pues el Poseedor es un argumento adicional en la cláusula. Este tipo de posesión es común en las lenguas del mundo, siendo un fenómeno que sirve para satisfacer las necesidades comunicativas de los hablantes. Hay diversos tipos de construcciones de PE: (i) construcciones que codifican R como objeto indirecto y $\mathrm{D}$ como objeto directo; (ii) construcciones con incorporación nominal; (iii) aplicativización de R que implica su promoción como argumento; (iv) codificación de $\mathrm{R}$ como objeto, sin dativo, incorporación nominal ni aplicativo; $\mathrm{y}$ (v) codificación de R y casos de doble sujeto (Payne y Barshi, 1999).

En el caso del Poseído, este puede perder su estatus argumental si es incorporado al verbo. También puede ser desviado y operar como un oblicuo, o podría perder su función, convirtiéndose en parásito (estatus que la gramática relacional llama chômeur), incluso conservando las marcas de objeto o sujeto. Este elemento no puede funcionar como dativo, objeto indirecto u objeto primario, porque esas son las relaciones que asume típicamente el Poseedor (Payne y Barshi, 1999).

En un plano más explicativo, como observamos, la posesión nominal se constituye en base a una relación semántica muy cercana entre los elementos (v.gr., parte-todo, parentesco, propiedad). En los casos en que los componentes requieren ser individuados o puestos en primer plano, la construcción posesiva nominal se escinde, estableciéndose la posesión externa o la "separación del Poseedor" (Aikhenvald, 2013: 36-39). En ella puede ser que el Poseedor no forme parte de una construcción nominal junto al Poseído, sino que "se promueve" a un rol sintáctico de argumento del verbo. La PE puede implicar relaciones de holonimia o de parentesco, o algún otro tipo de relación estrecha entre $\mathrm{R}$ y $\mathrm{D}$.

Así, en estas construcciones $\mathrm{R}$ puede ser tratado como una entidad separada, lo que generalmente implica que ha sido significativamente afectado por la acción expresada por el predicado. La motivación principal de que $\mathrm{R}$ sea considerado un argumento es la necesidad de individualizarlo, por lo que comienza a operar en 
primer plano, mientras D aparece en un segundo plano. R ocupa un lugar alto en la jerarquía nominal, pues prototípicamente es animado o humano (Aikhenvald, 2013: 40). En (15) se presenta el contraste en castellano entre la posesión nominal y la posesión externa: en (a) la PN va encabezada por un pronombre posesivo en función R, en tanto el sustantivo opera como D; por otro lado, en (b) se observa la promoción del Poseedor a objeto indirecto me (afectado), lo que va aparejado a un cambio en la valencia de la construcción, pasando de transitiva a ditransitiva. Finalmente, en (c) se observa que $\mathrm{R}$ se codifica simultáneamente como dativo y como pronombre posesivo en la PN.

(15) Castellano, familia indoeuropea

a. Juan quebró mi taza. (PN)

b. Juan me quebró la taza. (PE)

c. Juan me quebró mi taza. (PE+PN)

Aikhenvald (2013) señala que la incorporación nominal tiene un efecto similar al de la posesión externa, ya que en estos casos igualmente se escinde la relación posesiva entre $\mathrm{R}$ y $\mathrm{D}$, en tanto que $\mathrm{R}$ asciende en estatus y $\mathrm{D}$ es puesto en segundo plano. La incorporación de D se limitaría a casos de posesión inalienable (especialmente a partes del cuerpo).

\section{LA POSESIÓN EN LAS GRAMÁTICAS DEL MAPUZUGUN}

Antes de ingresar al análisis de los tipos de posesión existentes, presentamos una revisión de los desarrollos de este tópico en las gramáticas del mapuzugun. Esta tarea se realizará a través de un seguimiento histórico del tema en las obras producidas.

En su obra de 1684, Luis de Valdivia reconoce un caso genitivo (v.gr. chaoñi 'del padre') y presenta los "pronombres posesivos" del mapuzugun, distinguiendo los números singular, dual y plural. Además, indica que los posesivos "son los genitivos enteros de todos los pronombres primitivos" (v.gr. inche ñi, eimi mi), aunque "bastará poner las partículas que añaden los genitivos sobre el nominativo" (v.gr. ni, mi) (Valdivia, 1684: 11).

En Febrés (1846) se exponen los pronombres primitivos de la lengua; aquí la caracterización del sistema posesivo se muestra en función de: (a) el caso genitivo para los posesivos inche ñi ('de mí') y (b) el pronombre posesivo "como mío, tuyo... son el genitivo entero del primitivo ó sola su partícula, guardando el correspondiente numero" (Febrés, 1846: 13). Febrés (1846) agrega que las marcas de genitivo anteponen "palabras de adorno" como ta (tañi, tami, taiñ). Havestadt 
(1883) presenta un análisis similar al de Febrés (1846); basándose en la idea de genitivo, agrega la regla "genitivus plerumque pracedit substantivum ad quod pertinet ${ }^{\prime \prime}$ (Havestadt, 1883: 18). La Grasserie (1898: 15) aborda el tema de la posesión siguiendo los mismos patrones ya expuestos: reconoce un caso genitivo (chao-ñ $i$ 'padre su' para el singular y pu-chao-ñi 'los padres su' para el plural) y analiza los pronombres posesivos como los genitivos de los pronombres de caso nominativo (La Grasserie, 1898: 19).

Un análisis más sutil es el realizado por Augusta (1903), quien ya en la lección I de su gramática muestra el uso del verbo nien 'tener, poseer' junto con el pronombre personal, lo que constata que el mapuzugun tiene un verbo especializado para expresar la posesión predicativa. Luego, en la lección III presenta el paradigma de los pronombres posesivos (cuadro 1), señalando las siguientes reglas: (a) los pronombres son invariables; (b) las formas $\tilde{n} i$ 'mío/a(s)' y $\tilde{n} i$ 'suyo/a(s)' pueden conducir a la ambigüedad, la cual se clarifica al explicitar el pronombre personal que precede al posesivo; y (c) los pronombres usan con mucha frecuencia la partícula $t a$.

Tabla I.

\begin{tabular}{|c|c|c|c|}
\hline Persona & Singular & Dual & Plural \\
\hline 1 & $\tilde{n} i^{\prime} \mathrm{mi}(\mathrm{s})^{\prime}$ & $y u$ 'nuestro/a(s)' & $i \bar{n}^{\prime}$ nuestro/a(s)' \\
\hline 2 & $m i^{\prime} \mathrm{tu}(\mathrm{s})^{\prime}$ & mu 'de ustedes' & mün 'de ustedes' \\
\hline 3 & & $\tilde{n} i^{\prime} \mathrm{su}(\mathrm{s})$ ' & \\
\hline
\end{tabular}

Finalmente, Augusta (1903) da cuenta de una distinción entre los esquemas de posesión nominal del mapuzugun. Se trata de un tema relevante que retomamos en los análisis posteriores; por el momento mencionamos lo que Augusta (1903) plantea en su descripción: "Si el sustantivo modificativo [D] denota posesión ó pertenencia respecto del modificado [R], como en las frases: 'la casa de Pedro'... en este caso usan el posesivo de la tercera persona ' $\tilde{n} i$, su' de una manera que españolizada dice: padre su casa/chau (ta) ñi ruka; su casa padre/ñi ruka (ta) chau” (Augusta, 1903: 22). Además, agrega:

Si el sustantivo modificativo señala una cosa, p. ej. las olas del mar, no usan del posesivo por lo regular, sino que juntan los dos sustantivos inmediatamente de manera que la parte precede al todo, el material de que una cosa está hecha, á la

6 "El genitivo generalmente precede al sustantivo al cual pertenece". 
cosa misma; v.gr.: 'la oreja de caballo', pilun kawellu [...] Pero el continente precede al contenido; v.gr.: 'la gente de las ciudades', waria che [...], 'una botella de cerveza' kiñe fotella cerveza [...] Si el sustantivo modificativo no representa el todo del modificado ni el material ni el contenido, ó, si lo indica, viene el mismo por un pronombre ó adjetivo ú otro complemento con la preposición "de", toman otra vez la construcción con el posesivo ñi; v.gr.: el largo del río, leufü ñi wif (Augusta, 1903: 22-23).

Por último, Augusta (1903) indica que en los casos en que el sustantivo modificado "expresa un influjo sobre el modificativo", es decir, una relación de poder o ascendencia sobre el otro término, se yuxtaponen ambos sin más (v.gr. gen ruka 'el dueño de la casa'; ñidol mapu 'el jefe del país'). Tanto la terminología como el análisis del trabajo de Moesbach (2013) siguen a Augusta (1903).

Para Smeets (2008: 133-138), en la frase nominal posesiva el Poseedor precede al Poseído, y entre ambos se inserta un pronombre correferente con el Poseedor (chaw ñi wenüy 'el amigo del padre', lit. 'padre su amigo'). Por otro lado, Smeets (2008) presenta la frase nominal genitiva, en la cual el objeto referido por el primer nombre de la frase forma parte, pertenece o está conectado con el referente de la segunda parte de la frase (namun mesa 'pata de la mesa').

También Salas (2006: 100) ilustra el paradigma de los posesivos y adopta la idea de que estos pueden aparecer precedidos y "reforzados" por la partícula ta $y$, adicionalmente, por el pronombre personal respectivo (eymi ta mi kawellu 'tu caballo', lit. "tú tu caballo"). Este uso concurrente del pronombre resuelve la ambigüedad entre $\tilde{n} i$ 'mi' y $\tilde{n} i$ 'su' (ver Tabla I).

Harmelink (1996) agrega un análisis de la estructura de la "frase posesiva", que corresponde a la posesión nominal. Su descripción incluye el paradigma de los pronombres posesivos, e instruye sobre la formación de las frases posesivas, considerando el orden de aparición de los elementos en mapuzugun y su contraste con el castellano. Adicionalmente, este autor describe la yuxtaposición de sustantivos y la formación de sustantivos compuestos. Presenta los siguientes ejemplos: küna ruka 'casa hecha de totora' (lit. 'totora casa') y rüme ruka 'casa hecha de junquillo' (lit. 'junquillo casa'); en estos casos, el autor indica que el primer sustantivo "califica” al segundo. Además, propone otros casos en que, en sus palabras, el primer elemento es parte o elemento del segundo ( $k a l$ ufisa 'lana de oveja').

Adelaar \& Muysken (2004) enfatizan la importancia gramatical que tienen los posesivos, a los que llama "modificadores posesivos", los que pueden preceder sustantivos o verbos no finitos, y cuya presencia es obligatoria en las construcciones posesivas. Además, indica que este "modificador" puede estar precedido por el elemento $t a$ "sin un cambio de significado notorio" (p. 519). También reconoce que el modificador posesivo puede ser precedido por un pronombre personal independiente que cumple el papel de énfasis o desambiguación, especialmente en 
el caso de tañi, que puede corresponder a la primera persona singular o a la tercera persona. Finalmente, mientras en dichas construcciones posesivas el modificador precede al modificado (tüfachi wentru ñi ruka 'la casa de este hombre', lit. 'este hombre su casa'), Adelaar \& Muysken (2004) señalan que en aposiciones donde la relación genitiva no está explícitamente marcada, el orden común es modificadomodificador (me yene 'ámbar', lit. 'excremento ballena'). Estas últimas construcciones tienen una interpretación parte-todo.

Zúñiga (2006) realiza una comparación entre el sistema posesivo del castellano y el del mapuzugun, presenta las reglas básicas de uso de estos elementos y agrega una nota comparativa sobre tipología de los posesivos que, en parte, inspira este nuevo estudio.

Finalmente, las gramáticas de Catrileo (2010) y Loncon (2011) también desarrollan el tópico de la posesión. Catrileo(2010) muestra los posesivos en el marco del esquema de posesión nominal y agrega que "el pronombre personal pospuesto también indica posesión” (p. 113). Por su parte, Loncon (2011: 59-62) señala que el adjetivo posesivo puede ser utilizado para marcar preposición de pertenencia; es decir, asigna caso genitivo. Agrega, además, que el uso del pronombre personal si bien es opcional, sirve para eliminar la ambigüedad entre la primera y tercera persona.

\section{LA INVESTIGACIÓN}

Los objetivos de esta investigación son: (1) dar cuenta de la codificación de la posesión en la lengua mapuche, y (2) presentar una tipología de las estructuras posesivas del mapuzugun hablado en el territorio de la Araucanía, Chile. Para lograr estos propósitos, se aplicó una prueba lingüística de construcciones posesivas a dos hablantes bilingües competentes en mapuzugun. Los entrevistados se adscriben al territorio de Maquehue, distante a 20 kilómetros de Temuco en dirección suroeste, zona donde se habla la variante "mapuche central", según Salas (2006). Para obtener la información se elicitaron construcciones posesivas a partir de la aplicación de una lista de cincuenta y dos estructuras, orientadas a explicitar las tres formas de posesión: nominal, predicativa y externa ${ }^{7}$. Dentro de la posesión nominal no se abordó la posesión pronominal, pues el tema, como se ha mostrado en la sección precedente, ha sido tratado por todas las descripciones de la lengua. El instrumento se aplicó a cada uno de los hablantes participantes. Además, se contó con la participación de cuatro hablantes competentes con quienes se discu-

${ }^{7}$ Los autores reconocen ciertas limitaciones respecto del instrumento utilizado. Este es válido para relevar los esquemas prototípicos de la posesión. Otra cosa implica trabajar con textos orales espontáneos, cuestión que se proyecta en trabajos posteriores. 
tieron las estructuras elicitadas ${ }^{8}$. El análisis se orienta a determinar cuáles son los esquemas prototípicos vigentes que enuncian los hablantes y los tipos de posesión producidos por los participantes.

Finalmente, hemos optado por la nomenclatura propuesta por Dixon (2010): el Poseedor se abrevia como R y el Poseído como D. Por otro lado, para la posesión predicativa tomamos la propuesta de Stassen (2013), pues es más específica y particularmente clara. Con estos elementos generaremos la descripción de las posesivas encontradas en nuestro corpus.

\section{LA DESCRIPCIÓN DE LAS ESTRUCTURAS Y DISCUSIÓN}

\subsection{Posesión nominal}

Este análisis se proyecta desde la indagación de las tres relaciones de posesión nucleares recurrentes en la perspectiva inter-lingüística: (A) relación de propiedad, (B) relación parte-todo y $(\mathrm{C})$ relaciones de parentesco 9 . La primera pregunta que nos hacemos es: ¿tiene el mapuzugun estrategias diferenciadas para expresar A, B y C?

En nuestra exploración encontramos que la lengua es sensible a estos factores, pues hay diferencias en la construcción posesiva nominal cuando se expresan estas relaciones. En (16) se observa una relación de propiedad (A), pues se establece que el Poseído es de propiedad del Poseedor:

$\begin{array}{lll} & \mathrm{R} & \mathrm{D} \\ \text { Hector } & \tilde{n} i & \text { kawell } \\ \text { Héctor } & 3 . \mathrm{R} & \text { caballo } \\ \text { 'el caballo de Héctor' (lit. 'Héctor su caballo') }\end{array}$

La estructura de la frase muestra que el orden de los elementos en la construcción es [R D]. Para expresar relaciones de parentesco (C), la construcción sigue el mismo esquema:

$\begin{array}{lll} & \mathrm{R} & \mathrm{D} \\ \text { Kuan } & \tilde{n} i & \text { zeya } \\ \text { Juan } & 3 . \mathrm{R} & \text { hermana }\end{array}$

'la hermana de Juan' (lit. 'Juan su hermana')

8 Agradecemos los conocimientos inestimables de pu kimeltuchefe José Catrileo, Gerardo Chandía, Ana Nanculef y Héctor Painequeo, quienes tienen como lengua materna el mapuzugun.

${ }^{9}$ Las relaciones que derivan de este núcleo, y que por lo tanto son subsidiarias, son: atributiva, locativa u orientacional y asociativa. 
Por otro lado, la relación parte-todo (B) se codifica a través de una construcción con el orden opuesto, como puede verse en (18):

$\begin{array}{llll}\mathrm{D} & \mathrm{R} & \mathrm{D} & \mathrm{R} \\ \text { pichuñ } & \text { ünüm } & \text { trülke } & \text { sañwe } \\ \text { pluma } & \text { pájaro } & \text { cuero } & \text { cerdo } \\ \text { 'pluma de pájaro' (lit. 'pluma pájaro') } & \text { 'cuero de cerdo' (lit. 'cuero cerdo') }\end{array}$

Para corroborar la existencia de estas dos estructuras, compárese los casos a y b de (19), y analícese (20):

(19) a.

$\begin{array}{llll} & \mathrm{R} & \mathrm{D} & \mathrm{b} . \\ \text { waka } & \tilde{n} i & \text { lichi } \\ \text { vaca } & 3 . \mathrm{R} & \text { leche } \\ \text { 'la leche de la vaca'(lit. 'vaca su leche') } \\ \text { ta } & \tilde{n} i \quad \text { chagül kuwü } \\ \text { he } & \mathrm{ENF}^{10} & 1 \text { sG.R } & \text { dedo mano } \\ \text { dedo de la mano' (lit. 'yo mi dedo mano') }\end{array}$

(20) iñche

$1 \mathrm{SG}$ 'mi dedo de la mano' (lit. 'yo mi dedo mano')

Observamos que hay diferencias estructurales evidentes entre las construcciones (a) y (b) de (19), las que pueden inferirse del tipo de relación posesiva básica que establecen. En el primer caso se trata de la relación de propiedad (A), es decir, 'la leche le pertenece a una vaca', mientras que en el segundo caso, la relación posesiva que se establece es parte-todo. Observamos también que el orden de los elementos en las construcciones es distinto, en (a) se da [R D] y en (b) [D R]. En la Tabla II se agrupan las relaciones nucleares y las construcciones que exhibe el mapuzugun para la posesión nominal. Obsérvese que, a diferencia de lo que ocurre en otras lenguas, las partes del cuerpo monomorfémicas (p.ej. 'cabeza') se comportan como Poseídos alienables en mapuzugun: Juan ñi logko 'la cabeza de Juan' y Juan ñi ruka 'la casa de Juan' son ejemplos de la misma construcción. Algunas partes del cuerpo (p.ej. logko moyo 'pezón', lit. 'cabeza de pecho') son bimorfémicas y muestran una estructura interna de posesión inalienable, al tiempo que como unidad exhibe una estructura alienable respecto del Poseedor (p.ej. fey ñi logko moyo 'el pezón de él/ella', lit. 'él/ella su cabeza pecho').

${ }^{10}$ Para efectos de nuestro análisis, seguiremos la propuesta de Salas (1992:100), quien señala que la partícula ta opera como enfatizador. Estamos conscientes de la discusión actual acerca del estatus funcional exacto de dicho elemento. 
Tabla II.

\begin{tabular}{|c|c|}
\hline \multicolumn{2}{|c|}{ Tipo de relación } \\
\hline Alienable: Propiedad (A) y parentesco $(\mathrm{C})$ & Inalienable: Parte-todo (B) \\
\hline Pedro ñi ${ }_{\mathrm{R}} r u k a_{\mathrm{D}}$ 'la casa de Pedro' & ilo $z a n ̃ w e$ 'carne de cerdo' \\
\hline Juan ñi ${ }_{\mathrm{R}} \tilde{n} u k e_{\mathrm{D}}$ 'la madre de Juan' & kal ${ }_{\mathrm{D}}$ ficha ${ }_{\mathrm{R}}$ 'lana de oveja' \\
\hline Juan $\tilde{n} i_{\mathrm{R}} \log k o_{\mathrm{D}}$ 'la cabeza de Juan' & $\begin{array}{l}\operatorname{logko}_{\mathrm{D}} \text { moyo }_{\mathrm{R}} \text { 'pezón' (lit. 'cabeza } \\
\text { de pecho') }\end{array}$ \\
\hline
\end{tabular}

Como ya se dijo, la relación parte-todo sigue el principio de iconicidad, ya que la estrecha dependencia entre los referentes se expresa por medio de una construcción sin marca posesiva. Sabemos que este principio opera como fundamento de la distinción entre posesión alienable e inalienable; la posesión inalienable generalmente presenta menos marcas formales en la construcción que la alienable, la que tiende a ser expresada por medio de estructuras analíticas. Estamos entonces frente a un caso de posesión inalienable para la relación parte-todo.

Siguiendo la tipología de Aikhenvald (2013), diremos que el mapuzugun presenta dos estrategias para la posesión nominal. La relación alienable se expresa mediante una marca genitiva (un adjetivo/pronombre posesivo que precede al Poseído); la referencia del Poseedor puede ser explicitada adicionalmente con un nombre común o propio o enfatizada/focalizada con un pronombre personal, ambos antepuestos a los otros dos elementos. La relación inalienable codifica la relación parte-todo por medio de la yuxtaposición de $\mathrm{D}$ y $\mathrm{R}$, en este orden.

Como vimos, Augusta (1903) llamó la atención sobre este tipo de posesión inalienable y la describió en función de principios muy cercanos a los tratados en esta descripción. Por otro lado, Harmelink (1996) también notó este fenómeno, pero lo presentó como una estructura calificativa, donde el primer elemento califica al segundo, del mismo modo como en mapuzugun el adjetivo precede al sustantivo que modifica. Sin embargo, desde nuestro análisis puede decirse que hay una relación de posesión básica que implica la parte y el todo, en la que el primer elemento es parte constituyente del segundo. De manera similar, Baker y Fasola (2009: 971-974) identifican la productividad de la composición en mapuzugun, identificando un tipo de compuesto cuyo núcleo se ubica a la izquierda y se caracteriza por ser un sustantivo relacional que toma al segundo sustantivo como su argumento, expresando relaciones parte-todo o contenedor-contenido. Esta misma idea es planteada por Zúñiga (2014), quien señala la existencia de dos tipos de expresiones compuestas: aquellas de núcleo final y aquellas de núcleo inicial, estas últimas están restringidas semánticamente a la relación parte-todo. 


\subsection{Posesión predicativa}

El análisis sobre la posesión predicativa seguirá la tipología propuesta por Aikhenvald (2013), recurriendo además a las funciones argumentales desempeñadas por los elementos de la construcción posesiva predicativa. Como fue expuesto arriba, existen tres estrategias formales empleadas para expresar la posesión predicativa: (i) construcciones con verbos de propiedad (incluyendo aquellos como 'tener' $\mathrm{R}=\mathrm{A}$ y $\mathrm{D}=\mathrm{O}$ y aquellos como 'pertenecer' $\mathrm{R}=\mathrm{O}$ y $\mathrm{D}=\mathrm{A}$ ); (ii) construcciones copulativas y cláusulas sin verbo, y (iii) construcciones con derivaciones posesivas (donde la posesión se expresa mediante afijos derivacionales).

En mapuzugun existe una construcción especializada para la posesión predicativa, encabezada por el verbo nien 'tener', el cual actúa como núcleo de la cláusula y concuerda en persona y número con el sujeto. Esta construcción corresponde a la primera estrategia en Aikhenvald (2013): construcciones con verbos de propiedad; y al primer tipo (y primer subtipo) en Stassen (2013): posesión con verbo especializado, equivalente a 'tener'. En (21) se aprecia el funcionamiento de este elemento:

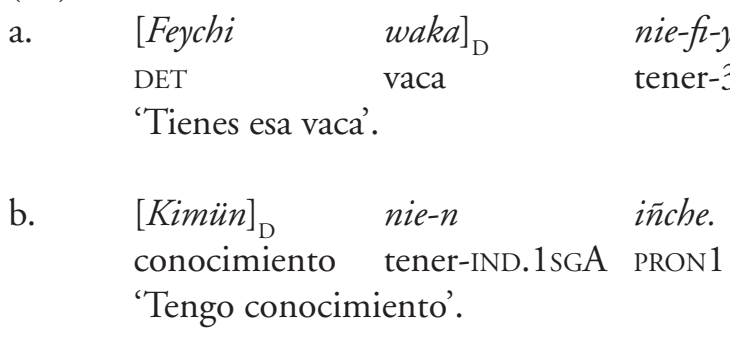

En el lexema verbal nie- se indexa el argumento A, que opera como Poseedor; el argumento $\mathrm{O}$ se expresa a través de un sintagma nominal (waka 'vaca'). Alternativamente, cuando el argumento $\mathrm{O}$ es definido, es posible también indexarlo al verbo, como se aprecia en (21a), por medio del morfema $-f$.

El orden habitual de los constituyentes de la predicativa posesiva es OVS. La estructura funciona como una construcción transitiva $\left[\mathrm{O}_{\mathrm{D}}+\right.$ nie- $\left.\mathrm{A}_{\mathrm{R}}\right]$. En cuanto al tipo de relación posesiva que se expresa con esta construcción, se observa que puede ser de propiedad (A) o de relaciones de parentesco (C), como se muestra en (22):

$$
\begin{array}{ll}
{\left[\begin{array}{ll}
\text { Ep } & \text { chuchu }
\end{array}\right]_{\mathrm{O}}} & \text { nie-y. } \\
\text { dos abuela } & \text { tener-IND[3A] } \\
\text { 'Tiene dos abuelas'. } &
\end{array}
$$


Los ejemplos (23a) y (24a) ilustran una codificación distinta: el sustantivo que expresa al Poseído se verbaliza con el morfema dependiente -ge, 'ser' y el sujeto expresa al Poseedor:

$$
\begin{aligned}
& \begin{array}{ll}
\text { Kure }_{\mathrm{D}}-\text { ge-y } & \text { Kuan }_{\mathrm{R}} . \\
\text { esposa-ser-IND[3] } & \text { Juan }
\end{array} \\
& \text { 'Juan tiene esposa / está casado'. (lit. 'Juan es (con) esposa') }
\end{aligned}
$$

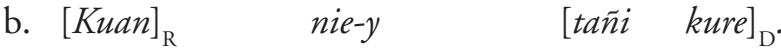

$$
\begin{aligned}
& \text { Juan tener-IND[3] 3R esposa } \\
& \text { (lit. 'Juan tiene su mujer') }
\end{aligned}
$$

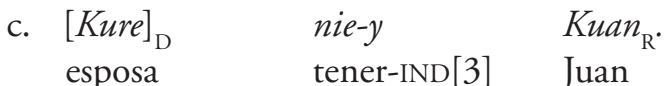

$$
\begin{aligned}
& \text { (lit. 'Juan tiene mujer') }
\end{aligned}
$$

La construcción utilizada en (23a) es similar a la que se utiliza en mapuzugun para codificar un tipo de construcción ecuativa (24a) y a la voz pasiva (24b). En casos como (23a) se utiliza para indicar un tipo de posesión que puede ser entendida como una propiedad permanente, generalmente inalienable, del Poseedor, lo que explica el uso de la misma estructura utilizada en oraciones como (24a).

(24) a. Weche-wentru-ge-y

joven-hombre-ser-IND[3] Juan

'Juan es un hombre joven'.
b. Mütrüm-ge-y
Kuan.
llamar-PAS-IND[3]
Juan
'Juan fue llamado / llamaron a Juan'.

Cabe destacar que la estructura con el verbalizador posesivo - ge, a diferencia de la estructura con el verbo nien, no corresponde al primer tipo de Aikhenvald (2013), sino al tercero, el de la verbalización posesiva. Así, el verbo resultante de la verbalización del sintagma nominal (v.gr. (23a): kure-ge-y) no corresponde a un verbo transitivo, sino a una estructura relacionada con las construcciones copulativas, que en algunos casos pueden tener una lectura existencial (25):

$$
\begin{aligned}
& {[\mathrm{Kal}]_{\mathrm{D}}-\mathrm{ge-y} \quad[\mathrm{ufcha}]_{\mathrm{R}} \text { ' }} \\
& \text { lana-ser-IND [3] oveja } \\
& \text { 'La oveja tiene lana / la oveja es lanuda'. (lit. 'la oveja es (con) lana') }
\end{aligned}
$$




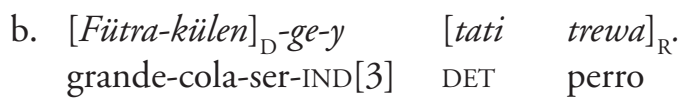
'El perro tiene cola larga'. (lit. 'el perro es (con) cola')

c. $[\text { Fütra-ke-ge }]_{\mathrm{D}}-g e-y \quad[\text { tati } \quad \text { pichi-che }]_{\mathrm{R}}$. grande-PL-ojo-ser-IND[3] DET pequeño-persona 'El niño tiene ojos grandes'. (lit. 'el niño es (con) ojos grandes')

Por lo tanto, se observa que en los casos en que el Poseído se conceptualiza como parte o propiedad permanente, preferentemente inalienable, en el mapuzugun hay disponibles construcciones alternativas (23 y 25), algunas de las cuales (23b-c, 25b-c) siguen un patrón de constituyentes equiparable al castellano, equivalentes a lo mostrado en (21). Mientras el primer tipo de estructura (con 'tener') presenta al Poseído y al Poseedor en sintagmas nominales diferentes, lo que enfatiza la interpretación de la manipulación de una entidad por la otra, el segundo tipo de estructura (verbalización posesiva) no presenta al Poseído como entidad diferente, sino como propiedad o rasgo del Poseedor. Concordantemente, con entidades no humanas y especialmente no animadas, sería inaceptable el uso de las estructuras predicativas con verbo nien (26 y 27):

(26) a. [Challwa $]_{\mathrm{D}}-$ ge-y $[t i \quad \text { lewfii }]_{\mathrm{R}}$. pez-ser- IND[3] DET río

‘El río tiene peces / hay peces en el río'. (lit. 'está con peces el río’)

b. $[\text { Tapül }]_{\mathrm{D}}-g e-y \quad$ ta $\quad[p u \quad \text { anümka }]_{\mathrm{R}}$. hoja=ser- IND[3] DISC PL planta(s)

'Las plantas tienen hojas'. (lit. 'están con hojas las plantas')

c. $[\text { Tromü }]_{\mathrm{D}}-g e-y \quad[\text { antü }]_{\mathrm{R}}$.

nube-ser-IND[3] día

'Tiene nubes el día / hay nubes'. (lit. 'está nuboso el día, está con nubes el día')

(27) a. ${ }^{*}[\mathrm{Ti} \quad \text { lewfii }]_{\mathrm{R}}$ nie-y $\quad[\text { challwa }]_{\mathrm{D}}$.

ART río tener-IND[3] pez/peces

'El río tiene peces'.

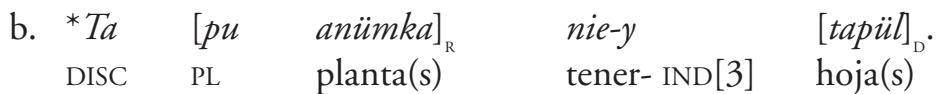

'Las plantas tienen hojas'. 


$$
\begin{array}{lll}
\text { c. }{ }^{*}[\text { Antü }]_{\mathrm{R}} & \text { nie-y } & {[\text { tromü }]_{\mathrm{D}} .} \\
\text { día } & \text { tener-IND [3] } & \text { nube }(s) \\
\text { 'El día tiene nubes'. } &
\end{array}
$$

Como se ve, la naturaleza del Poseedor y del Poseído es relevante para establecer las relaciones de posesión predicativa. Hasta el momento, la posesión predicativa transitiva (con 'tener') se utiliza cuando $\mathrm{R}$ es animado y $\mathrm{D}$ corresponde a objetos, animales, nociones abstractas y relaciones de parentesco. La verbalización posesiva también puede utilizarse cuando el Poseído corresponde a objetos, animales, y ciertas relaciones de parentesco, por lo que la diferencia entre ambas estructuras parece radicar tanto en la naturaleza del Poseedor como en la relación entre ambos. Con todo, en algunos casos ambas construcciones están disponibles (23). Respecto a esto, en la actualidad parece haber una tendencia a su codificación por medio de las estructuras analíticas $(23 \mathrm{~b}-\mathrm{c})$, aunque el respaldo o falseo de esta hipótesis requiere datos de uso.

En resumen, el mapuzugun tiene un verbo especializado para expresar la posesión predicativa. Se trata del verbo transitivo nien, que se usa con sustantivos que denotan objetos de la naturaleza y culturales, animales y relaciones de parentesco. Las relaciones de posesión básicas que se pueden expresar con este verbo son las de propiedad y parentesco. La posesión parte-todo puede expresarse con este verbo, aunque también puede hacerse, prototípicamente, marcando el sustantivo en función D con el verbalizador posesivo - ge, del mismo modo que se codifican la relación de un Poseedor con propiedades permanentes, preferentemente inalienables.

\subsection{Posesión externa}

La posesión externa (PE) no se infiere del sentido del verbo. Aquí, el Poseedor aparece separado del Poseído, lo que se traduce en una re-organización de los papeles semánticos. Según lo anterior, el papel semántico de R se codifica en un constituyente separado de $\mathrm{D}$, como un argumento del verbo, mientras que $\mathrm{D}$ puede mantener su estatus o codificarse como un adjunto. A nivel estructural, el Poseedor no está en la misma frase del Poseído (Álvarez, 2012; Payne y Barshi, 1999). Por otro lado, la estrategia de incorporación nominal también puede considerarse una forma de posesión externa, ya que la relación sintagmática original entre $\mathrm{R}$ y $\mathrm{D}$ se escinde igualmente (Payne y Barshi, 1999; Aikhenvald, 2013).

La pregunta correspondiente en esta parte del análisis es si en la lengua mapuche existen construcciones del tipo Juan me quebró la taza, distintas de construcciones como Juan quebró mi taza. Esta interrogante está relacionada con los medios que provee el mapuzugun para modificar la valencia verbal, entre los que 
se cuentan los morfemas aplicativos y la incorporación nominal. Así, por ejemplo, tenemos:

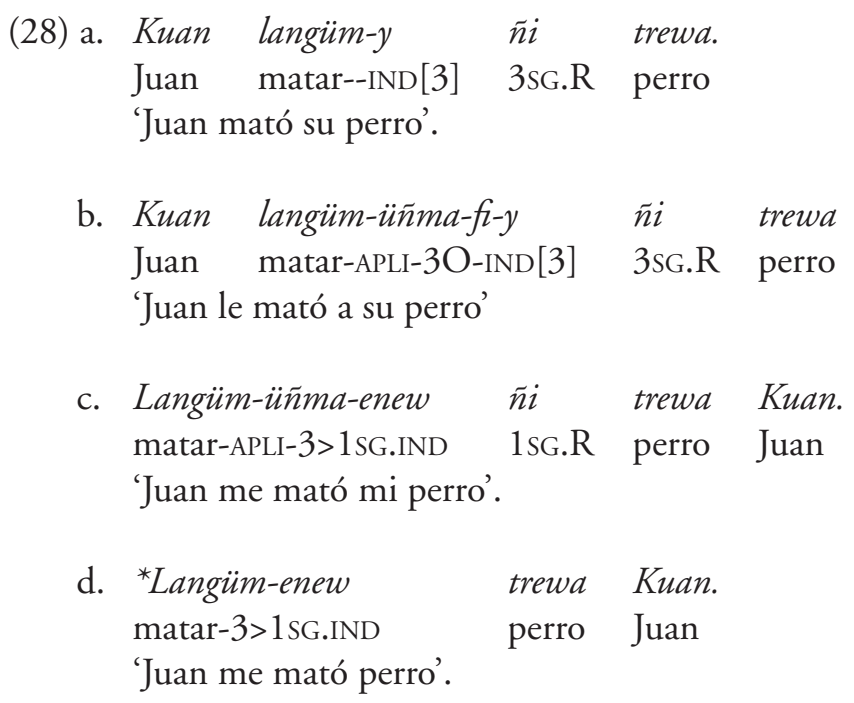

En los casos en que 'el perro' se conceptualiza como propiedad de un Poseedor, la construcción, en su forma directa, se da en (28a) y (28b); sin embargo, en (28b) el poseedor aparece más afectado que en (28a). En (28a) el sintagma nominal ñi trewa funciona como paciente directo del evento (objeto primario base), que por efecto de la aplicativización (28b), es relegado a un estatus de objeto aplicado. La aplicativización implica que el afectado, en este caso, el dueño del perro (una tercera persona $-f i$ ) adquiere el estatus de objeto primario. Por otro lado, en (28c) se muestra otra PE con aplicativización. En esta construcción inversa, Kuan es el objeto agente, ñi trewa es el paciente que se recodifica como objeto aplicado, emergiendo el Poseedor como sujeto paciente (primera persona 'yo'). Se observa que el mecanismo morfológico de la inversión recae sobre el sujeto gramatical (primera persona singular, en este caso), interpretado semánticamente como un paciente indirecto en la oración, separando $\mathrm{R}$ y $\mathrm{D}$ para redistribuir el primero y darle estatus argumental.

Si se compara (28a) con (28b) y (28c), se observa que la valencia del verbo langümün 'matar' cambia. Tanto (28b) como (28c) son trivalentes, lo que se produce por la aplicativización dada por -( $\ddot{u}) \tilde{n} m a$. Este proceso permite observar dos elementos que son propios de una PE: (1) la promoción de R (Poseedor) con la marcación aplicativa y (2) su separación de D (Poseído), que mantiene su relación sintáctica de objeto con el verbo, pero en un segundo plano. Este elemento es relevante a la hora de examinar la estrategia de PE del mapuzugun, ya que su uso 
involucra un incremento de la valencia verbal y la re-organización tanto de los argumentos verbales como de la relación de posesión establecida en la estructura eventiva.

El caso (28d) no es aceptable en mapuzugun. En este se observa la ausencia del aplicativo y del adjetivo/pronombre posesivo de la frase nominal (ñi). Por un lado, en ausencia del aplicativo el sujeto gramatical sería interpretado como paciente directo (i.e. 'Fui asesinado por Juan') y, por otro, la marcación del Poseedor es obligatoria.

En (29a) se observa el uso de una forma directa que contiene una frase posesiva, si se compara con (29b) pueden notarse las diferencias que tiene la construcción respecto de una que tiene PE:

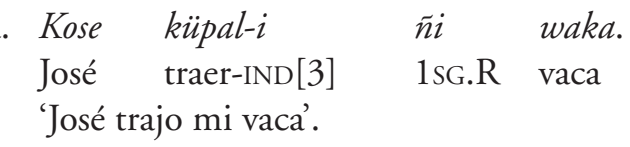

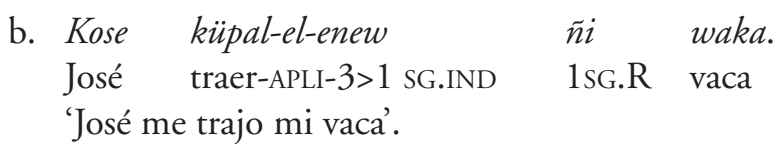

Las diferencias entre estas construcciones radican en la aplicativización de (29b) a través del morfema -(l)el, lo que introduce un argumento nuevo en la estructura argumental, aumentando la valencia verbal y reorganizando los papeles semánticos. Además, nótese que la primera persona singular es al mismo tiempo un argumento del verbo küpalün 'traer' y un elemento constitutivo (R) de la frase nominal ñi waka en (29b).

El morfema aplicativo -(l)el opera como un marcador semántico de beneficio por defecto, que se opone a $-\tilde{n} m a$, el cual se ha descrito como un morfema no marcado con función de beneficio o detrimento (Zúñiga y Kittilä, 2010) o un morfema que indica afectación (Golluscio, 2010). Cuando el verbo tiene un sentido que naturalmente implicará un perjudicado, se emplea la construcción ilustrada en (30):

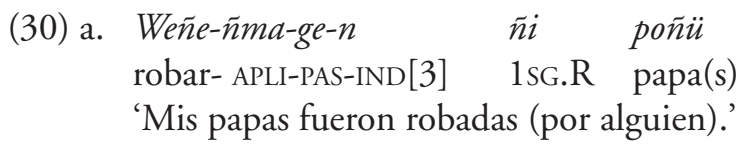

b. Weñe-ñma-enew ñi poñü.

robar-APLI-3>1 SG.IND 1SG.R papa(s)

'Me robó / robaron mis papas'. 
Nuevamente, si comparamos las construcciones (30a) y (30b), observamos que lo que tienen en común es el aplicativizador $(-\tilde{n} m a)$; sin embargo, la diferencia radica en que la aplicativización de (30a) recae sobre $\tilde{n} i$ poñi, por un lado, y la presencia del morfema - ge indicando que una persona indeterminada realizó la acción. En (30b), en tanto, vemos que hay una reorganización de los papeles semánticos en la estructura eventiva siguiendo el patrón ya exhibido en (29b) y (28c).

En síntesis, la PE en mapuzugun se canaliza por medio de un proceso de aplicativización que reorganiza los papeles semánticos del evento y aumenta la valencia del verbo. Los aplicativos -ñma y -(l)el también pueden funcionar como malefactivo y benefactivo, respectivamente. En cuanto a la estrategia de incorporación nominal (IC) como alternativa para expresar la PE, dado que el mapuzugun tiene productividad en IC, también puede aplicarse por medio del mismo recurso aplicativo.

\section{REFLEXIONES FINALES}

Las escuelas tipológicas reseñadas en este trabajo son complementarias y todas útiles para lograr una descripción más potente y pormenorizada del fenómeno de la posesión en el mapuzugun. La descripción lingüística que se ha desarrollado hasta aquí nos conduce al establecimiento de la siguiente clasificación tipológica del mapuzugun:

En cuanto a la posesión nominal, siguiendo los lineamientos complementarios reseñados en la introducción (Bickel y Nichols, 2013; Nichols y Bickel, 2013a y 2013b; Aikhenvald, 2013 y Dixon, 2010), puede decirse que el mapuzugun es sensible a la relación alienabilidad vs. inalienabilidad, esta última para la posesión que implica relación parte-todo y derivados. La estrategia que utiliza para codificar esta relación es la yuxtaposición o aposición. La relación alienable se aplica sobre relaciones de propiedad (incluidas las partes del cuerpo) y parentesco, codificándose esta por medio de un adjetivo/pronombre posesivo que antecede al Poseído (véase Tabla II).

En segundo lugar, adoptando la tipología de Aikhenvald (2013), existen dos tipos de construcciones de posesión predicativa: una con un verbo transitivo especializado (nien), que expresa relaciones posesivas de propiedad y parentesco, y otra con la verbalización del elemento Poseído mediante un sufijo derivacional (-ge), utilizada cuando el Poseído se conceptualiza como propiedad permanente y relativamente inalienable del Poseedor (incluyendo algunos casos de relaciones parte-todo). La segunda construcción es utilizada especialmente en casos en que el Poseedor tiene un bajo grado de manipulación del elemento Poseído, ya sea porque este último es un rasgo del primero y/o porque el primero es una entidad inanimada. 
En tercer lugar, siguiendo a Payne y Barshi (1999), el mapuzugun muestra construcciones de posesión externa, las que se realizan a partir de la aplicativización de un verbo, hecho que produce un cambio o reasignación de valencias sobre el núcleo eventivo.

Finalmente, con este trabajo se enriquece nuestro conocimiento de las lenguas surandinas, permitiéndonos situar al mapuzugun en el contexto de la tipología de estructuras posesivas. Los datos presentados pueden ser útiles para el establecimiento de relaciones lingüísticas areales y, sin duda, confirman la importancia y utilidad del marco tipológico para la descripción, análisis y comprensión del funcionamiento de las lenguas naturales.

\section{REFERENCIAS}

Adelaar, Willem y Muysken, Pieter. (2004). The Languages of the Andes. Cambridge, England: Cambridge University Press.

Aikhenvald, Alexandra. (2013). "Possession and ownership: A cross-linguistic perspective". En A. Aikhenvald \& R. M. W. Dixon. (eds.). Possession and Ownership: A cross-linguistic typology. (pp. 1-57). Oxford, England: Oxford University Press.

Aikhenvald, Alexandra. (2015). The Art of Grammar: A practical guide. Oxford, England: Oxford University Press.

Álvarez, Albert. (2012). "Estructuras posesivas en yaqui". UniverSOS, 9, 115-132. Álvarez, Albert y Muchembled, Fany. (2015). "Verbos posesivos en taracahita: esquemas fuentes y rutas evolutivas". Amerindia, 37, 97-120.

Álvarez, José. (1996). "Construcciones posesivas en guajiro”. Opción, 19, 21-44. Augusta, Félix. (1903). Gramática Araucana. Valdivia: Imprenta Central J. Lampert.

Baker, Mark y Fasola, Carlos. (2009). “Araucanian: Mapudungun”. En R. Lieber \& P. Štekauer. (eds.). The Oxford Handbook of Compoundin (pp. 594-608). Oxford, England: Oxford University Press.

Bickel, Balthasar y Nichols, Johanna. (2013). "Obligatory possessive inflection". En M. S. Dryer \& M. Haspelmath. (eds.) The World Atlas of Language Structures Online. Leipzig, Germany: Max Planck Institute for Evolutionary Anthropology. Disponible en http://wals.info/chapter/58, Accessed on 2017-0528.

Catrileo, María. (2010). La lengua mapuche en el siglo XXI. Valdivia, Chile: Universidad Austral de Chile.

Dixon, Robert. (2010). Basic Linguistic Theory, Vol. 2: Grammatical topics. Oxford, England: Oxford University Press. 
Febrés, Andrés. (1846). [1765]. Gramática de la lengua chilena. Santiago de Chile, Chile: Imprenta de los Tribunales.

Fernández Garay, Ana. (2004). "Aspectos semántico-cognitivos de la posesión en tehuelche". UniverSOS, 1: 43-60.

Golluscio, Lucía. (2010). Ditransitive constructions in Mapudungun. En A. Malchukov, M. Haspelmath y B. Comrie. (Ed.), Studies in ditransitive constructions. A comparative handbook (pp. 710-756). Berlín/Nueva York, Alemania/ Estados Unidos: Walter de Gruyter.

Harmelink, Bryan. (1996). Manual de aprendizaje del idioma mapuche. Aspectos morfológicos y sintácticos. Temuco, Chile: Ediciones Universidad de La Frontera.

Haspelmath, Martin. (2008). "Alienable vs inalienable possessive constructions". En Leipzig Spring School on Linguistic Diversity. Disponible en https://www. eva.mpg.de/lingua/conference/08_springschool/pdf/course_materials/Haspelmath_Possessives.pdf

Havestadt, Bernardo. (1883). [1775]. Chilidúgíu sive Tractatus linguae chilensis. Leipzig, Germany: B. G. Teubner.

Heine, Bernd. (1997). Possession: Cognitive Sources, Forces and Grammaticalization. Cambridge, England: University Press.

La Grasserie, Raoul de. (1898). "Langue Auca (ou langue indigène du Chili). Grammaire, dictionnaire, textes traduits et analyses". París, France: J. Maisonneuve.

Loncon, Elisa. (2011). Morfología y aspectos del mapudungun. México, México: Universidad Autónoma Metropolitana.

Moesbach, Ernesto. (2013) [1963]. Idioma Mapuche. Santiago de Chile, Chile: RIL.

Muchembled, Fany. (2014). La posesión predicativa en lenguas yutoaztecas. Tesis doctoral, Institut National des Langues et Civilisations Orientales.

Nichols, Johanna y Bickel, Balthasar. (2013a). "Locus of marking in possessive noun phrases". En M. S. Dryer \& M. Haspelmath. (eds.). The World Atlas of Language Structures Online. Leipzig, Germany: Max Planck Institute for Evolutionary Anthropology. Disponible en http://wals.info/chapter/24, Accessed on 2017-08-25.

Nichols, Johanna y Bickel, Balthasar. (2013b). Possessive classification. En M. S. Dryer \& M. Haspelmath. (eds.). The World Atlas of Language Structures Online. Leipzig, Germany: Max Planck Institute for Evolutionary Anthropology. Disponible en http://wals.info/chapter/59, Accessed on 2017-05-28.

Oquendo, Luis (2007). "La construcción posesiva en Japreria". Synergies, 3, 157172.

Pamies, Antonio (2004). "La posesión en lenguas americanas". UniverSOS, 1, 81102. 
Payne, Doris y Barshi, Immanuel (eds.) (1999). External Possession. Amsterdam, Netherland: John Benjamins.

Salas, Adalberto. (1992). El mapuche o araucano: fonología, gramática, y antología de cuentos. Madrid, España: Editorial MAPFRE.

Salas, Adalberto. (2006). El mapuche o araucano. Santiago, Chile: Centro Estudios Públicos.

Smeets, Ineke. (2008). A Grammar of Mapuche. Berlin, Germany: Mouton de Gruyter.

Stassen, León. (2009). Predicative Possession. Oxford: Oxford University Press.

Stassen, León. (2013). "Predicative possession”. En M. S. Dryer \& M. Haspelmath. (eds.). The World Atlas of Language Structures Online. Leipzig: Max Planck Institute for Evolutionary Anthropology. Disponible en http://wals. info/chapter/117, Accessed on 2017-08-16.

Valdivia, Luis. (1684). Arte y gramática general de la lengua que corre en todo el reyno de Chile, con un vocabulario, y confesionario. Sevilla, España: Thomas López de Haro.

Zúñiga, Fernando. (2006). Mapudungun. El habla mapuche. Santiago de Chile, Chile: Centro de Estudios Públicos.

Zúñiga, Fernando. (2014). "Nominal compounds in Mapudungun”. En S. Danielsen; K. Hannss \& F. Zúñiga (eds.). Word Formation in South American Languages (pp. 11-31). Amsterdam, Netherland: John Benjamins.

Zúniga, Fernando y Kittilä, Seppo. (eds.). (2010). Benefactives and Malefactives: Typological perspectives and case studies. Amsterdam, Netherland: John Benjamins. 